\section{Patients preferences concerning ultrasound guided regional anesthesia}

\author{
N.M. Freitas, E. Semedo, A. Marques, R. Orfao, \\ J.M. Nunes \\ Department of Anesthesiology, University of Coimbra \\ Hospitals, Coimbra, Portugal
}

Background and Aims: Ultrasound guided regional anesthesia is usually presented as a safer and more accurate technique for the Anesthesiologist, especially considering peripheral nerve blocks. We would like to know also the patients perspective, pre-operatively and postoperatively, concerning the use of ultrasound probe to ad the regional anesthesia procedures, and if there is, or not, any difference on their overall confidence and satisfaction.

Methods: As part of the preoperative informed consent we asked 40 patients ASA I-II scheduled electively for regional anesthesia about their preference on adding ultrasound (primary end point). Then we randomized 2 groups of 20 patients each for regional technique with guided ultrasound and without guided ultrasound asking postoperatively for their satisfaction considering the anesthetic procedure (primary end point). We have checked for success rates, intraoperative and postoperative complications ( $<12$ hours) of both groups (secondary end point).

Results: Pre-operatively 27 patients $(67.5 \%)$ preferred adding ultrasound to regional anesthesia neurostimulators and 13 patients $(32.5 \%)$ had no preference at all. None refused. Increment of safety, previous positive experience with ultrasound and increment of comfort and confidence were the main reasons pointed out for the positive option. Results concerning postoperative questionnaires are still being collected.

Conclusions: So far, we can conclude that a significant majority of patients' preferred pre-operatively the use of ultrasound guided regional anesthesia for peripheral nerve blocks.

\section{The use of fascia iliaca compartment blocks to provide analgesia for fractured neck of femur in the emergency department}

\author{
G. Foxall1', D. Esberger², P. Miller², R. Freij ${ }^{2}$, J. Varcoe'2, \\ N. Bedforth ${ }^{1}$ \\ 'Department of Anaesthesia, University Hospital, Queen's \\ Medical Centre, Nottingham, UK, ${ }^{2}$ Department of \\ Emergency Medicine, University Hospital, Queen's \\ Medical Centre, Nottingham, UK
}

Background $\boldsymbol{\varepsilon}$ Aims: Neck of femur fracture (NOF) is a common presentation to UK emergency departments (ED). High mobilisation pain scores and concerns about opioid-induced side effects can lead to suboptimal pain relief in this group. The fascia iliaca compartment block (FICB) can provide pre-operative analgesia in these patients, with particular efficacy for pain on movement (1).

Methods: A prospective audit of 90 patients presenting to the ED with suspected NOF who were all given FICB. ED medical staff performed the blocks using a $22 \mathrm{G}$ short-bevelled $50 \mathrm{~mm}$ Plexufix needle (Braun Melsungen AG) and bupivacaine $(0.25 \%) 0.5 \mathrm{~mL}$ $\mathrm{kg}-1$ up to a maximum of $40 \mathrm{~mL}$. Variables recorded included pain scores at rest and on passive movement and grade of ED doctor performing the block.

Results: A convenience sample of 90 patients were included (mean weight 64kgs; $78 \%$ female; $52.5 \%$ intra-capsular; $47.5 \%$ extra-capsular). ED consultants or registrars performed $71 \%$ of blocks. A "double pop" was detected in $90 \%$ of cases. Mean volume of local administered was $30 \mathrm{~mL}$. The block reduced pain to 'mild' or 'nil' from 'moderate' or 'severe' in 59\% of patients at rest and $60 \%$ on movement. The block failed to achieve any reduction in pain in $11 \%$ of patients at rest and $16 \%$ on movement after 30 minutes. Simple linear regression analysis revealed no association between grade of staff and pain reduction after adjustment for initial pain score. No difference in efficacy was found when comparing analgesia for intra-capsular vs. extra-capsular fractures. There were no reports of vessel puncture, nerve damage or signs and symptoms of local anaesthetic toxicity.

Conclusions: The FICB delivered a good standard of pain relief for NOF in the ED with no adverse outcomes.

Reference

1. Candal-Couto, J et al. Injury 2005; 36(4): 505-510. 